ARTIGO ORIGINAL ORIGINAL ARTICLE

Palavras-chave:

vacinas, custo-efetividade, influenza

\section{Custo-efetividade da vacina contra influenza quadrivalente baseada em células comparada com a trivalente baseada em ovo do Programa Nacional de Imunizações brasileiro}

\author{
Cost-effectiveness of cell-based quadrivalent versus \\ egg-based trivalent influenza vaccination in the \\ Brazilian National Immunization Program \\ Isabella Ballalai', João Toniolo', Renato Kfouri², Glaucia Vespa ${ }^{3}$, Cecilia Magneres ${ }^{4}$, \\ Joaquin Mould-Quevedo ${ }^{5}$, Bernardo Pires ${ }^{6}$, Rodrigo Angerami
}

DOI: 10.21115/JBES.v13.n2.p136-44

\section{RESUMO}

Objetivos: Este estudo teve como objetivo avaliar desfechos clínico-econômicos associados à vacina contra influenza quadrivalente baseada em células (QIVc) versus a vacina trivalente atualmente utilizada (TIVe) para prevenção sazonal de influenza no Programa Nacional de Imunizações (PNI) brasileiro. Métodos: Um modelo estático de árvore de decisão foi usado. Considerou-se um total de 54.071.642 indivíduos vacinados em 2019; a circulação de influenza por subtipo foi baseada em dados de vigilância epidemiológica. A efetividade da vacina (EV) TIVe foi extraída de metanálises publicadas; já a EV relativa da QIVc foi retirada de um estudo observacional retrospectivo. A incompatibilidade antigênica da vacina com vírus circulantes foi baseada em fontes retrospectivas internacionais. O uso de recursos baseou-se em estudos do mundo real. Custos unitários foram retirados de tabelas-padrão publicados em 2019 em reais (BRL). Resultados: Substituir a TIVe pela QIVc pode evitar, anualmente, casos sintomáticos (452.065) e reduzir visitas ambulatoriais (118.735), hospitalizações (15.466), mortes (2.753), custos médicos (-BRL 46.677.357) e custos indiretos (-BRL 59.962.135). O número anual de anos de vida ajustados por qualidade de vida (QALYs) pode aumentar em 96.129. Resultados de base a partir da perspectiva do pagador mostram uma razão de custo-efetividade incremental (RCEI) de BRL 17.293/QALY e, da perspectiva da sociedade, o RCEl obtido foi de um ganho de BRL 16.669/QALY. Usando o Produto Interno Bruto (PIB) brasileiro como um limiar (BRL 34.533/ QALY), trocar a TIVe pela QIVc no PNI pode ser uma estratégia altamente custo-efetiva. Conclusões: O uso da QIVc pelo PNI tem potencial para ser altamente custo-efetivo tanto da perspectiva do pagador quanto da sociedade.

\section{ABSTRACT}

Objectives: This study aimed to estimate health and economic outcomes associated to cell-based quadrivalent influenza vaccine (QIVC) versus current trivalent influenza vaccines (TIVe) for seasonal influenza prevention in the Brazilian National Immunization Program (NIP), from the societal and public payer perspectives. Methods: A 1-year static decision-tree model based on literature was used. 54,071,642 total vaccinated individuals in 2019 were considered; influenza subtype circulation was based on Brazilian epidemiologic data (2009-2019). TIVe vaccine effectiveness (VE) was

\footnotetext{
Recebido em: 09/03/2021. Aprovado para publicação em: 21/07/2021.

1. Sociedade Brasileira de Imunizações, São Paulo, SP, Brasil.

2. Sociedade Brasileira de Pediatria, São Paulo, SP, Brasil.

3. Seqirus Vaccines Ltd., São Paulo, SP, Brasil.

4. Seqirus S.A., Buenos Aires, Argentina.

5. Seqirus Vaccines Ltd., New Jersey, NJ, EUA.

6. Kantar Health, São Paulo, SP, Brasil.

7. Universidade Estadual de Campinas, Campinas, SP, Brasil.

Financiamento: Este estudo foi financiado pela Seqirus Vaccines Ltd., NJ, EUA

Instituição onde trabalho foi executado: Seqirus Vaccines Ltd. (São Paulo, Brasil) e Kantar Health Division (São Paulo, Brasil).

Autor correspondente: Joaquin Mould-Quevedo. Seqirus Vaccines Ltd. 25 Deforest Avenue, Summit, NJ 07901, Estados Unidos

da América. Telefone: +1 (908) 739-0174. Celular: +1 (908) 517-6456. E-mail: Joaquin.Mould-Quevedo@seqirus.com
} 
extracted from a published meta-analysis and QIVc relative VE from an international retrospective observational study. A/H3N2 egg-adaptation and B mismatch to recommended strain were gathered from international retrospective sources. Resource use was obtained from real-world studies. Inputs were adjusted to influenza subtype and multiple age groups with Brazilian literature. Unit costs were retrieved from published standard tables in 2019 Brazilian Reais (BRL). Results: Replacing TIVe with QIVc, can annually avert symptomatic cases $(452,065)$ and reduce outpatient visits $(118,735)$; hospitalizations (15,466), deaths (2,753), overall medical direct costs (-BRL 46,677,357) and indirect costs (-BRL 59,962,135). The annual number of quality-adjusted life-years (QALYS) could be increased by 96,129 . Base case results from the payer perspective show an incremental cost-effectiveness ratio (ICER) of BRL 17,293/QALY gained and from the societal perspective the ICER obtained was BRL 16,669/QALY gained. Using the Brazilian Gross Domestic Product (GDP) as a threshold (BRL 34,533/ QALY) switching TIVe with QIVc in the NIP can be a highly cost-effective strategy, leading to a high QALY increment and preventing medical and indirect costs. Conclusions: The use of QIVc by the NIP has the potential to be highly cost-effective in the payer and society perspective.

\section{Introdução}

A influenza sazonal é uma infecção respiratória aguda e estima-se que ela acarreta globalmente 650.000 mortes respiratórias e 5 milhões de casos severos por ano (luliano et al., 2018). Agregada à carga respiratória da doença, a influenza também é potencialmente responsável pelo desenvolvimento de infecções secundárias, agravamento de doenças subjacentes (WHO, 2015) e várias complicações extrapulmonares, como insuficiência renal aguda, encefalopatia, síndrome de Guillain-Barré, infarto do miocárdio e miocardite (Rothberg \& Haessler, 2010).

No Brasil, o Programa Nacional de Imunizações (PNI) distribui gratuitamente a vacina contra influenza para populações consideradas de risco desde 1999. Os grupos populacionais cobertos ampliaram-se continuamente ao longo dos anos para incluir crianças entre 6 meses e 6 anos, idosos (acima de 60 anos), mulheres grávidas e no pós-parto, populações indígenas, pacientes com doenças crônicas (incluindo pneumopatia, cardiopatia, nefropatia, hepatopatia, diabetes, doenças neurológicas, imunossupressão, obesidade e doenças hematológicas), trabalhadores do sistema de saúde e prisional, pessoas privadas de liberdade, professores e menores de idade em medidas educacionais (SVS, 2019c), tornando a vacinação contra influenza pelo PNI um programa de 54 miIhões de doses (Monteiro, 2019). Para 2020, após uma nova ampliação da cobertura para incluir adultos a partir de 55 anos ou mais, espera-se a distribuição de 68 milhões de doses (Monteiro, 2019).

Apesar da contínua ampliação da cobertura da população, a vacina contra influenza distribuída pelo PNI vem sendo a mesma nos últimos 20 anos (Taveira et al., 2019). Assim, o programa atualmente distribui vacinas contra influenza trivalentes baseadas em ovo (TIVe), fabricadas a partir de culturas monovalentes quimicamente inativadas de três vírus de influenza, incluindo $\mathrm{A} / \mathrm{H} 1 \mathrm{~N} 1, \mathrm{~A} / \mathrm{H} 3 \mathrm{~N} 2$ e uma única linhagem de influenza B (Taveira et al., 2019). Em relação à definição da cepa de influenza B recomendada para inclusão na vacina trivalente, trata-se de um evento complexo e sujeito a frequentes falhas: 45\% das temporadas entre 2001 e 2013. Em estudo retrospectivo brasileiro, foi sugerido que o uso da vacina quadrivalente contra influenza poderia ser altamente benéfico, levando-se em consideração a cocirculação. (Barros et al., 2016).

Adicionalmente, o processo de produção baseado em ovo pode levar a mutações adaptativas do vírus influenza devido à seleção em células aviárias, sobretudo em vírus de influenza A/H3N2. Um estudo retrospectivo evidenciou que, em 55\% das temporadas nos EUA entre 2002 e 2018, antígenos da influenza A/H3N2 em vacinas baseadas em ovo apresentaram $<25 \%$ de similaridade com os vírus circulantes. No mesmo período, tal reduzida similaridade antigênica foi encontrada em apenas $4 \%$ das temporadas com vacinas produzidas em células de mamíferos, sugerindo um potencial benefício elevado do uso da vacina baseada em célula (Rajaram et al., 2020).

Preocupações com a adaptação do antigênio e outras questões de fabricação da vacina baseada em ovo, tais como limitações do aumento da produção da vacina devido ao estoque de ovos férteis, contaminação imprevisível de ovos com vírus aviários e limitação do crescimento de algumas cepas, levaram ao desenvolvimento das vacinas baseadas em célula, que mostraram ter imunogenicidade e reatogenicidade satisfatórias, ao mesmo tempo mantendo uma produção flexível (Audsley \& Tannock, 2008; Rajaram et al., 2020; Zost et al., 2017).

Nesse contexto, o presente estudo visa estimar o custo-efetividade do uso da vacina contra influenza quadrivalente baseada em célula (QIVc) pelo PNI brasileiro, em comparação com o esquema atualmente vigente apenas com TIVe.

\section{Métodos}

\section{Estrutura do modelo}

Um modelo estático de árvore de decisão de um ano foi desenvolvido para estimar a vacinação, casos de influenza, complicações, o uso de recursos, ganhos de anos de vida ajustados por qualidade de vida (QALY), custos e óbitos. A estrutura do modelo foi baseada em um estudo de custo-efetividade publicado anteriormente no Brasil, que avaliou vários tipos de modelos econômicos (desde simples 
modelos estáticos até complexos modelos vitalícios de cadeia de Markov), todos resultando em desfechos clínicos e de custos esperados e similares entre todos para o cenário brasileiro (Van Bellinghen et al., 2018). Portanto, um modelo de árvore de decisão estático foi adotado em detrimento de um dinâmico. A estrutura do modelo é exibida na figura 1.

\section{Dados demográficos}

A população brasileira de 2020 de 211.755 .692 foi adotada, conforme reportado pelo Instituto Brasileiro de Geografia e Estatística (IBGE, 2018). A vacinação foi considerada para um total de 54.071.642 pessoas, cobrindo as populações no Brasil durante 2019 (SVS, 2019c). A população brasileira com comorbidades foi estimada em 21.995.255, baseada em dados do Ministério da Saúde e da Sociedade Brasileira de Pediatria de 2014 (Soespe, 2014).

\section{Epidemiologia da influenza}

A circulação sazonal de influenza no Brasil foi estimada com base em resultados de subtipos de cepa de pacientes com doenças similares a influenza reportados em relatórios epidemiológicos brasileiros entre 2009 e 2019 (SVS, 2019b). Apenas subtipos de influenza $A$ foram reportados em relatórios oficiais; portanto, os dados de circulação da linhagem de influenza B foram baseados em dados epidemiológicos brasileiros entre 2001 e 2013, conforme publicados em um estudo retrospectivo (Barros et al., 2016).

A probabilidade média para o período do nosso estudo para similaridade baixa ou sem antigênico entre a vacina baseada em ovo e os vírus influenza A/H3N2 circulante foi obtida do Centro Mundial de Influenza para as temporadas 2002-2003 a 2017-2018 nos hemisférios norte e sul, conforme publicado em um estudo retrospectivo (Rajaram et al., 2020).

\section{Cobertura da vacinação}

Apesar de a cobertura da vacinação de 2020 ter sido ampliada para incluir adultos mais velhos acima de 55 anos, não havia dados suficientes de taxas de cobertura para essa faixa etária. Portanto, a cobertura da vacina por idade foi baseada em dados reportados do PNI a partir de 2019 (SVS, 2019a), conforme exibido na tabela 1.

\section{Taxas de ataque de influenza}

As taxas de ataque de influenza em pacientes saudáveis não vacinados foram retiradas de um estudo de carga de doença nos EUA publicado em 2007. Assumiu-se que um total de

Tabela 1. População e cobertura da vacina no Brasil

\begin{tabular}{lcc}
\hline Faixa etária & $\begin{array}{c}\text { População brasileira } \\
\text { em 2020 }\end{array}$ & $\begin{array}{c}\text { Doses de TIVe } \\
\text { fornecidas pelo PNI } \\
\text { em 2019 (cobertura } \\
\text { da vacinação em } \\
\text { 2019) }\end{array}$ \\
\hline$<6$ anos & 17731728 & $14531499(81,9 \%)$ \\
\hline 6 a 17 anos & 35768410 & $3387645(9,4 \%)$ \\
\hline 18 a 49 anos & 104183396 & $11989859(11,5 \%)$ \\
\hline 50 a 59 anos & 23875081 & $2554355(10,9 \%)$ \\
\hline 60 a 64 anos & 9383728 & $6066790(66,7 \%)$ \\
\hline 65 a 79 anos & 16372296 & $11805594(75,0 \%)$ \\
\hline acima de 80 anos & 4441053 & $2890366(67,7 \%)$ \\
\hline
\end{tabular}

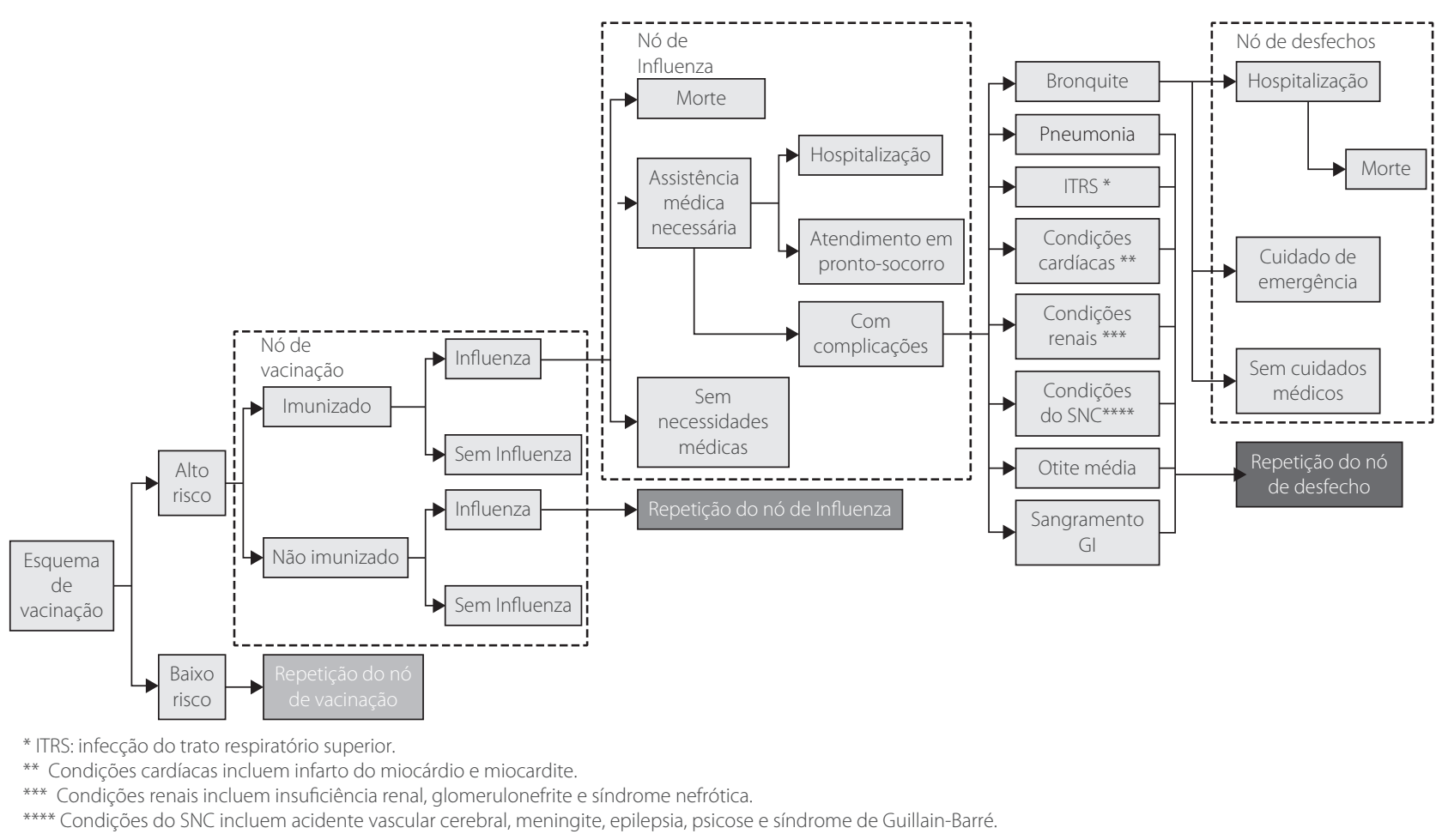

Figura 1. Estrutura do modelo de árvore de decisão. 
20,3\% de crianças abaixo de 5 anos, 10,2\% de crianças entre 5 e 17 anos, 6,6\% de adultos (18 a 64 anos) e 9,0\% de idosos (acima de 65 anos) contraiam influenza anualmente (Molinari et al., 2007)

\section{Efetividade da vacina}

A absoluta efetividade da vacina (aEV) TIVe contra influenza A foi baseada em metanálises publicadas de vacinação pediátrica (Jefferson et al., 2018), adulta (Demicheli et al., 2018b) e idosa (Demicheli et al., 2018a). A aEV da TIVe contra influenza A foi assumida como sendo de 64,0\% em crianças, de 59,0\% em adultos e de 58,0\% em idosos. Similarmente, a aEV da TIVe contra linhagens pareadas da influenza B foi assumida como sendo de 77,0\% em adultos, enquanto a proteção cruzada contra linhagens não pareadas foi assumida em 52,0\% em adultos.

O não pareamento da influenza B foi assumido como inexistente para QIV, como previamente reportado em outros estudos econômicos (Van Bellinghen et al., 2018).

A relativa efetividade da vacina ( $\mathrm{rEV}$ ) devido à similaridade baixa ou sem antigênico foi adotada para a influenza A/H3N2 com base em um estudo eletrônico de gráfico dos EUA. O estudo reportou uma rEV de 36,2\% [intervalo de confiança (IC) de 95\%: 26,1 a 44,9] em pessoas acima de 4 anos que receberam QIV baseada em célula ou em ovo durante a temporada de 2017-2018 (Boikos et al., 2020). Incluímos esse desfecho porque é a melhor referência que reflete a população geral contida em nosso modelo econômico com QIVc. Como a antigenicidade depende da sazonalidade, tal rEV foi assumida apenas para temporadas com similaridade baixa ou sem antigênico entre o vírus QIVe e o vírus circulante (Rajaram et al., 2020).

\section{Complicações relacionadas à influenza}

O risco de complicações em crianças e adultos devido à influenza foi baseado em estudos retrospectivos (Gums et al., 2008; Karve et al., 2013) e categorizado em sete grupos diferentes: bronquite, pneumonia, infecção do trato respiratório superior (ITRS), condições cardíacas (infarto do miocárdio e miocardite), condições renais (insuficiência renal, glomerulonefrite e síndrome nefrótica), condições do SNC (acidente vascular cerebral, encefalopatias, epilepsia, psicose e síndrome de Guillain-Barré) e otite média aguda. A categorização foi baseada em estudos econômicos anteriores sobre influenza (Dolk et al., 2016; Van Bellinghen et al., 2018).

\section{Desfechos clínicos}

As taxas de hospitalização e mortalidade por causa e faixa etária foram baseadas em dados brasileiros de hospitalização do mundo real.

A razão de probabilidade de hospitalização e todas as causas de morte em pacientes com comorbidades foram obtidas de uma metanálise publicada (Mertz et al., 2013).
Dados de qualidade de vida foram baseados em estudos populacionais nos EUA devido à falta de dados nacionais.

A expectativa de vida ajustada pela qualidade (QALE) em uma abordagem de idade de morte prematura foi adotada, conforme reportado em um estudo econômico anterior no Brasil para evitar uma simplificação excessiva dos benefícios vitalícios da vacinação contra influenza (Van Bellinghen et al., 2018).

\section{Custos da vacinação}

O preço médio por dose pago pelo Ministério da Saúde foi adotado para a TIVe (BRL 15,12 em 2019) (Brasil, 2020a). Apenas preços de mercado estavam disponíveis para a QIVc (BRL 46,73); portanto, empregaram-se análises de sensibilidade de preço (Agência Nacional de Vigilância Sanitária, 2020).

Com base nos preços padronizados da área da saúde no Brasil, uma taxa administrativa de BRL 0,63 foi acrescentada a todos os preços de vacina por dose (Brasil, 2020b).

\section{Custos de atendimento médico}

Os custos ambulatoriais foram baseados nos preços padronizados da área da saúde no Brasil (Brasil, 2020b). Os custos de hospitalização e tempo de permanência foram baseados no custo médio de hospitalização por causa em 2019, conforme reportado por bancos de dados de hospitais (Brasil, 2019).

\section{Custos indiretos}

O absenteísmo foi considerado tanto para adultos em idade ativa doentes (homens entre 18 e 65 anos e mulheres entre 18 e 60 anos) precisando de atendimento médico quanto para adultos em idade ativa acompanhando menores de idade doentes ao atendimento médico (INSS, 2018). Um dia inteiro de absenteísmo foi adotado para cada dia de hospitalização e meio dia de absenteísmo foi adotado para cada visita ambulatorial. As durações das hospitalização foram baseadas em tempos médios de hospitalização por influenza por faixa etária no Brasil em 2019 (Brasil, 2019).

Os custos de produção por morte prematura foram calculados com base na expectativa de vida na idade da morte prematura e idades ativas para homens e mulheres. O Produto Interno Bruto (PIB) brasileiro per capita [BRL 34.533 em 2019 (IBGE, 2020)] foi assumido como perda de produção por ano de trabalho perdido.

\section{Moeda}

Os custos foram calculados em reais (BRL) em 2019, calculado a USD 0,186, de acordo com o Banco Central do Brasil (Bacen, 2020). Nenhum desconto foi empregado devido à curta duração da análise, de acordo com as diretrizes brasileiras (Brasil, 2014).

\section{Análises de sensibilidade}

Modelos matemáticos são inerentemente cheios de incertezas advindas tanto do uso de dados de fontes diferentes e da variabilidade dos próprios dados (Briggs et al., 2006). Portan- 
to, análises de sensibilidade foram empregadas para estimar o impacto das incertezas nos resultados reportados.

Parâmetros epidemiológicos clínicos e econômicos foram testados nas análises de sensibilidade determinística e probabilística.

Nas análises de sensibilidade determinísticas, os resultados foram calculados com uma variação de 10\% sobre cada valor de parâmetro por meio de simulações de Monte Carlo de segunda ordem. O impacto do parâmetro sobre o resultado foi posteriormente classificado.

Em relação às análises de sensibilidade probabilísticas, todos os parâmetros foram simultaneamente variados de acordo com uma abordagem estocástica. Distribuições paramétricas foram assumidas para os seguintes parâmetros múltiplos (Briggs et al., 2006):

- Distribuição gama: apenas parâmetros positivos, como custos e qualidade de vida;

- Distribuição beta: parâmetros binomiais, como taxas, probabilidades e riscos;

- Distribuição normal: parâmetros grandes populacionais, como advindos de bancos de dados médicos e estudos de coorte;

- Distribuição lognormal: apenas parâmetros populacionais positivos, como risco relativo.

Um erro-padrão de 10\% foi assumido para os parâmetros sem desvio reportado.

Todos os parâmetros, seus valores, erros-padrão e distribuições assumidas estão disponíveis no conteúdo suplementar (Suplementar 2).

\section{Disposição em pagar (WTP)}

Devido a uma falta de limiar oficial de custo-efetividade adotado pela Comissão Nacional de Incorporação de Tecnologias no Sistema Único de Saúde (Conitec), o PIB per capita foi usado de acordo com a prática comum no país (Canuto, 2012).

Os resultados abaixo de um PIB per capita foram considerados como sendo altamente custo-efetivos (razão de custo- -efetividade incremental - RCEI $\leq B R L$ 34.533), os resultados entre uma e três vezes o PIB per capita foram considerados como sendo custo-efetivos (BRL $34.533<R C E I \leq B R L$ 103.599) e todos os resultados acima de três vezes o PIB per capita foram considerados como não sendo custo-efetivos (RCEl > BRL 103.599).

\section{Resultados}

\section{Base}

Substituir o TIVe, disponível na campanha anual de vacinação contra influenza, pelo QIVc para a população brasileira pode evitar, por ano, 452.065 casos sintomáticos, 118.735 visitas ambulatoriais, 15.466 hospitalizações e 2.753 mortes, levando a uma economia de BRL 46.677.357 e BRL 59.962.135 em custos médicos diretos e indiretos, respectivamente.

O uso do QIVc pelo preço de mercado levaria a um aumento de BRL 1.709.062.532 em custos de vacinação ao público pagador.

Considerando todos os desfechos clínicos, o número anual de QALYs aumentaria em 96.129, resultando em uma razão incremental de custo-efetividade (RCEI) de BRL 17.293/ QALY, obtida da perspectiva do pagador, e BRL 16.669/QALY, obtida da perspectiva da sociedade. Ambos os resultados de caso base ficam abaixo de 1 PIB per capita e, portanto, seriam considerados como altamente custo-efetivos.

\section{Análises de sensibilidade}

Ao considerar o preço relativo, o uso da QIVc é econômico até um aumento de preço de $6 \%$ da TIVe, na perspectiva do pagador, e um aumento de 13\%, na perspectiva da sociedade. O uso do QIVc também é altamente custo-efetivo até um aumento de preço de $412 \%$ da TIVe, na perspectiva do pagador, e um aumento de 419\%, na perspectiva da sociedade.

Apesar de o pareamento da influenza B ter baixo impacto nos resultados da QIVC, o pareamento da influenza A/H3N2 tem grande impacto. Como mostrado na figura 2, a vacinação por QIVc permanece como custo-efetiva até $90 \%$ do pareamento de $\mathrm{A} / \mathrm{H} 3 \mathrm{~N} 2$.
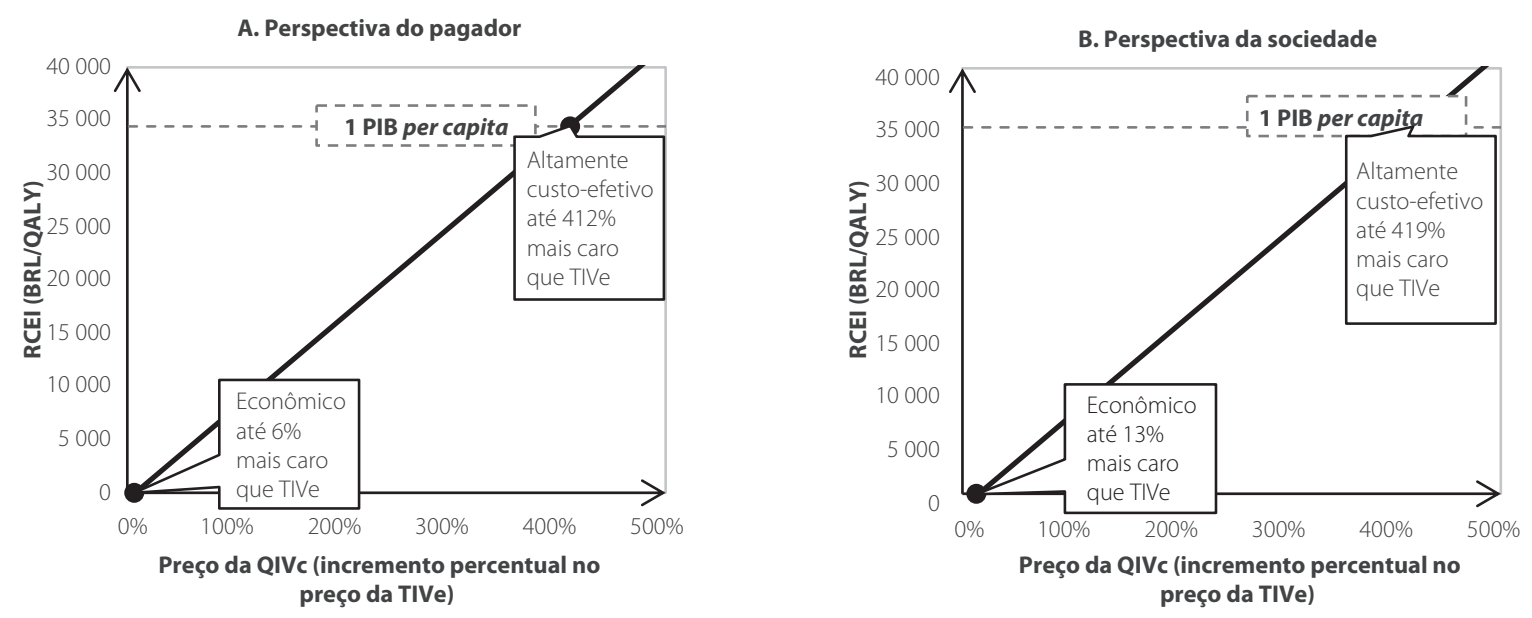

Figura 2. Análise do preço relativo da QIVc (incremento percentual no preço em relação ao preço de mercado da TIVe): (A) Perspectiva do pagador; (B) Perspectiva da sociedade. 
Efetividade da vacina, preço da vacina e circulação de influenza foram correlacionados à alta incerteza nos resultados, como mostrado na figura 3.

Ao considerar todas as fontes de incerteza simultaneamente, as perspectivas tanto do pagador quanto da sociedade ficam aproximadas, como mostrado na figura 4. Em ambas as perspectivas, o uso da QIVC tem 85\% de chance de ser custo-efetiva ( $\leq 3$ PIB per capita).

\section{Discussão}

A influenza apresenta um desafio de saúde pública relevante no mundo inteiro, tanto pela morbidade quanto pela mortalidade. Por esse motivo, estratégias e políticas de prevenção e controle devem ser continuamente reavaliadas quando confrontadas com novas evidências clínicas, epidemiológicas e econômicas. Nesse contexto, o modelo de decisão se tornou uma ferramenta importante para tomadores de decisão alcançarem os objetivos desejados no setor de saúde.

Resultados para o cenário apenas da TIVe foram comparados com dados do PNI do mundo real como uma validação para os resultados do modelo. As hospitalizações estimadas por influenza não complicada foram próximas aos dados reportados nos bancos de dados de hospitais brasileiros em 2018 (23.946 vs. 19.172) (Brasil, 2019). A taxa estimada de mor-
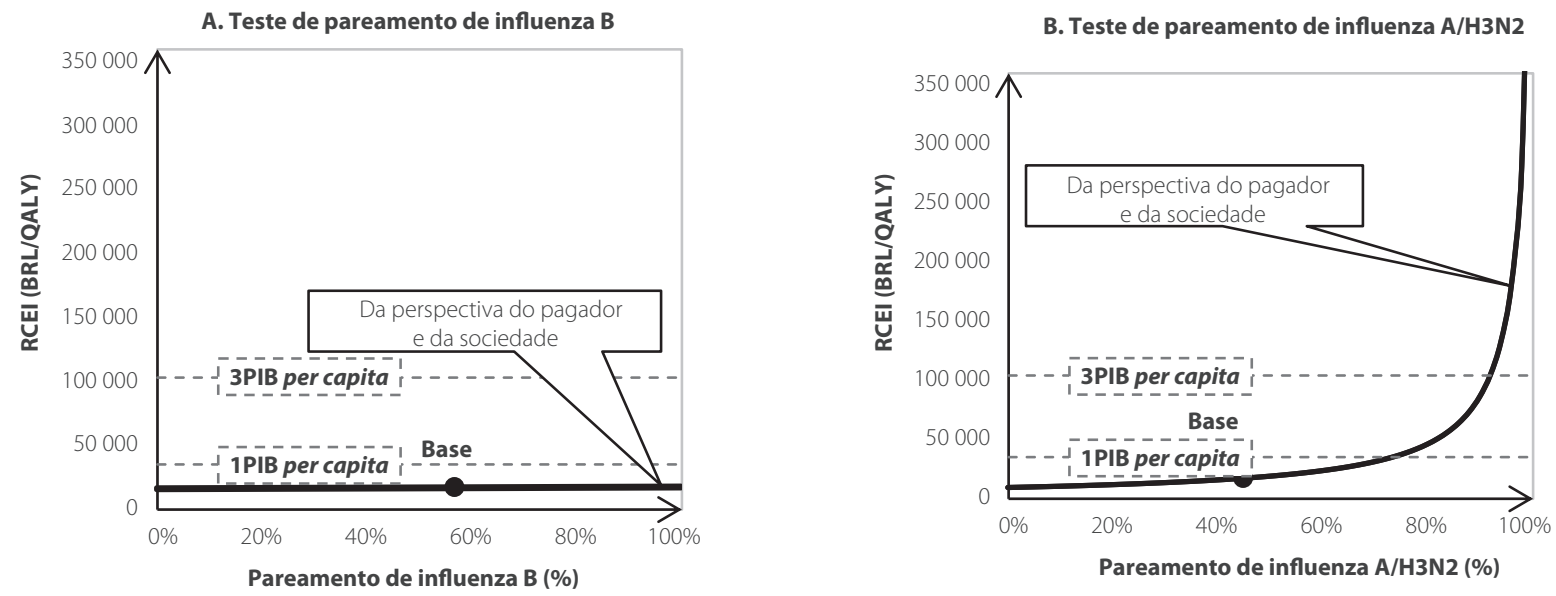

Figura 3. Análises de sensibilidade unidirecionais com cenários de pareamento: (A) Teste de pareamento de influenza B; (B) Teste de pareamento de influenza A/H3N2.

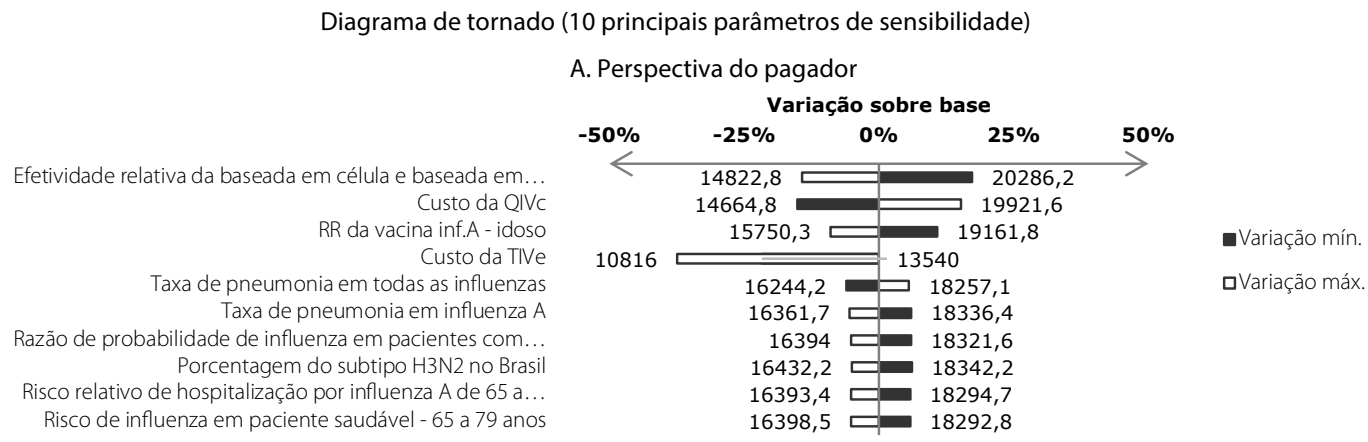

B. Perspectiva da sociedade

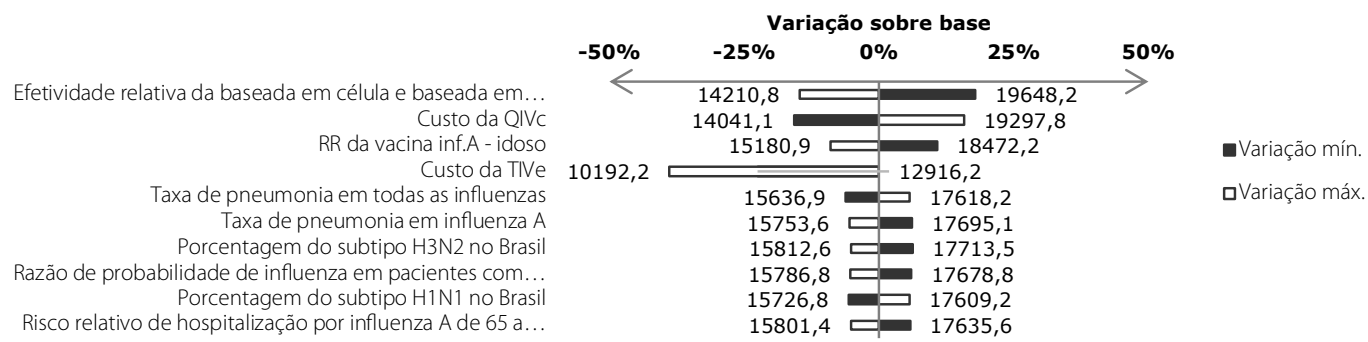

Figura 4. Diagrama de tornado dos resultados da análise de sensibilidade determinística: (A) Perspectiva do pagador; (B) Perspectiva da sociedade. 
talidade por influenza não complicada foi próxima da taxa de mortalidade no Brasil entre 2009 e 2019, como reportado por boletins epidemiológicos (1,19/100.000 vs. 0,09-1,07/100.000) (SVS, 2019b). Pela falta de robustos dados brasileiros sobre complicações primárias e secundárias relacionadas à influenza, não foi possível comparar a mortalidade estimada total; porém, como as taxas de hospitalização e mortalidade foram baseadas em dados de hospital do mundo real no Brasil, a variação deve ser mínima.

Apesar de o uso do QIVc pelo PNI ter sido previsto como custo-efetivo a preço de mercado, os preços do mundo real provavelmente são menores. Portanto, custos incrementais de vacinação reportados aqui empregando os preços máximos de mercado podem estar superestimados, devido ao impacto que tais parâmetros mostraram ter ao transmitir os resultados.

Análises econômicas internacionais publicadas anteriormente sobre a QIVc reportaram resultados similares de que o seu uso para a prevenção da influenza é potencialmente custo-efetiva na Espanha e Alemanha (Cai et al., 2020; Ruiz-Aragon et al., 2020).

Algumas limitações desse estudo incluem outros aspectos conhecidos da influenza que não foram empregados no modelo devido à ausência de dados quantitativos, como a perda funcional por hospitalização prolongada, descompensação metabólica e algumas complicações de idade pediátrica. A duração da hospitalização de idosos por influenza pode levar a aceleradas perdas musculares e de capacidade aeróbica (Mcelhaney, 2005). Foi reportado que pacientes com doenças metabólicas, doenças crônicas renais ou insuficiência renal sofreram descompensação metabólica temporária ou prolongada durante as infecções pelo vírus (McGuire et al., 2014). Por fim, a escassez de evidências nacionais em relação às taxas de ataque de influenza, efetividade da vacina e qualidade de vida na população brasileira representam um limite aos resultados reportados, já que os dados internacionais podem não ser consistentes com o cenário brasileiro.

\section{A. Perspectiva do pagador}
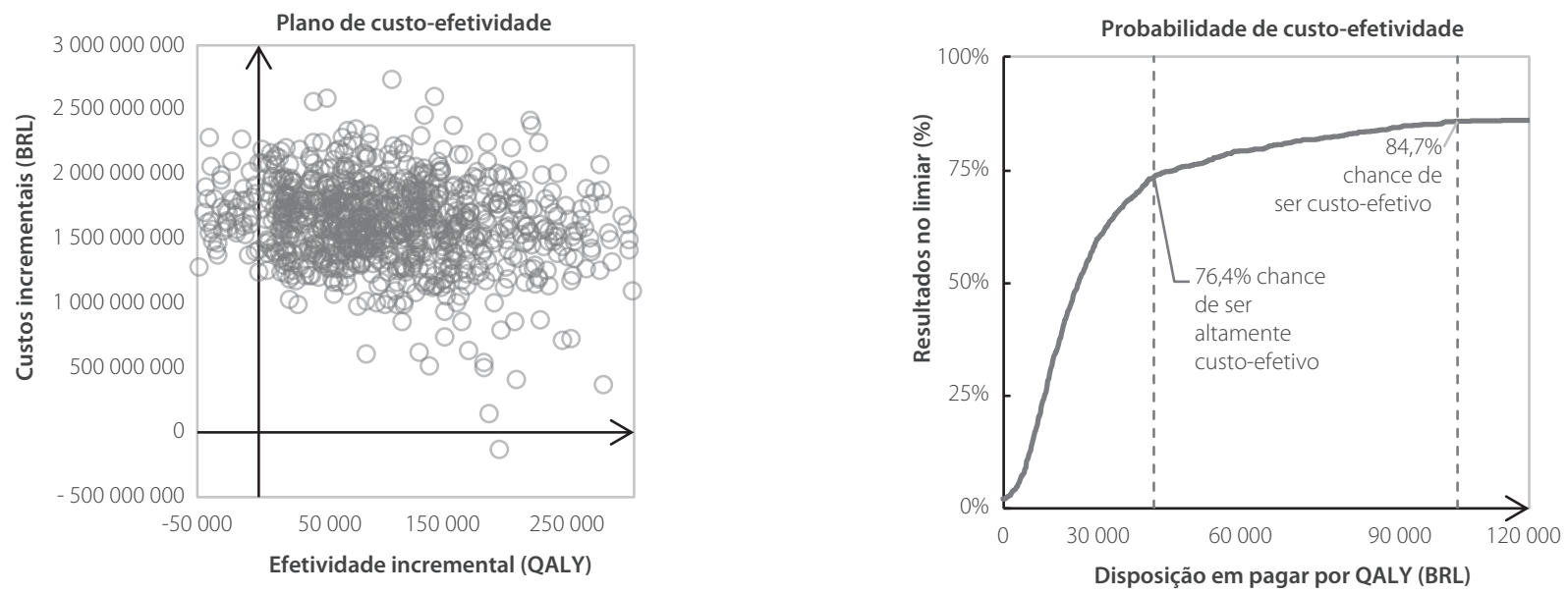

B. Perspectiva da sociedade
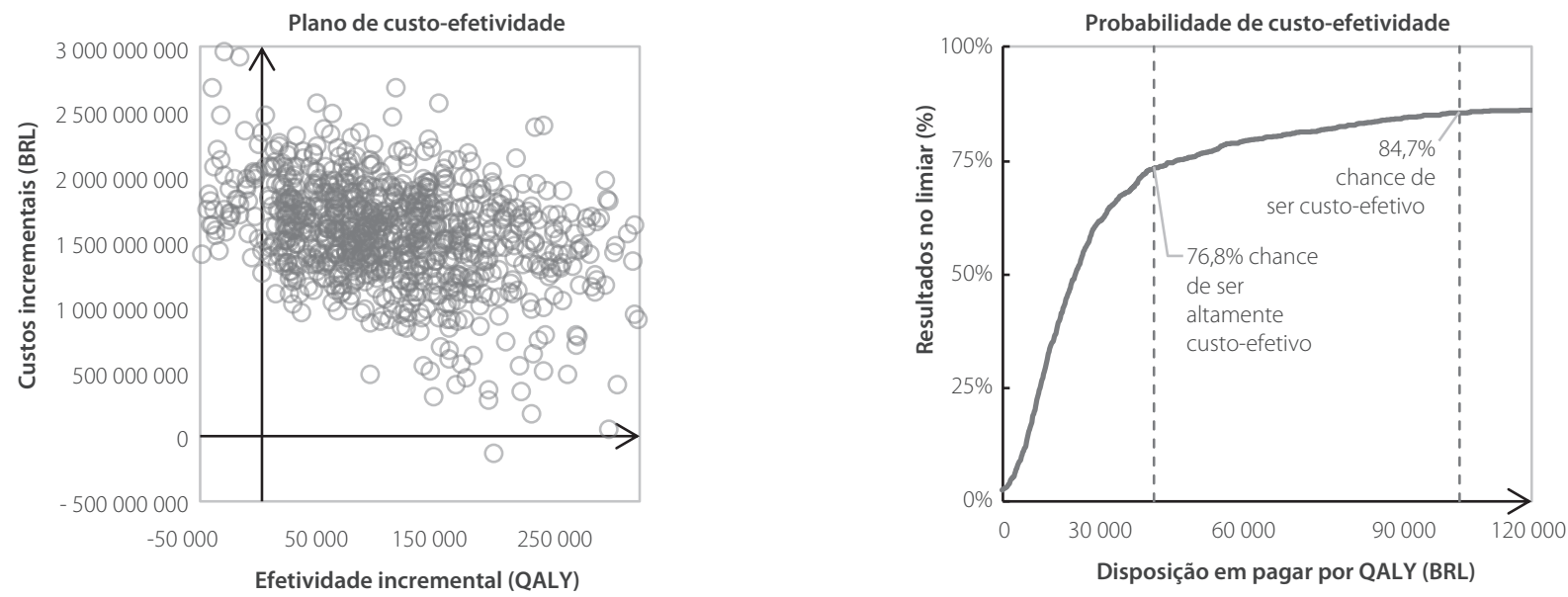

Figura 5. Análise de sensibilidade probabilística com 1.000 simulações: (A) Plano de custo-efetividade e limiar de probabilidade na perspectiva do pagador; (B) Plano de custo-efetividade e limiar de probabilidade na perspectiva da sociedade. 


\section{Conclusão}

Mesmo sem as ampliações de cobertura da população, o uso da QIVc pelo PNI tem potencial para ser altamente custo-efetivo tanto da perspectiva do pagador quanto da sociedade a preço de mercado, levando à prevenção de 118.735 visitas ambulatoriais, 15.466 hospitalizações e 2.753 mortes, resultando em um ganho de 96.129 QALY a BRL 17.293/QALY, da perspectiva do pagador, e BRL 16.669/QALY, da perspectiva da sociedade.

Ao considerar incertezas de parâmetro, os resultados da QIVc permanecem consistentes e até $86 \%$ das simulações probabilísticas foram custo-efetivas no limiar definido pela Organização Mundial da Saúde (OMS) de 3 PIB per capita.

\section{Agradecimentos}

A Mirian de Moura e Shanthy Krishnarajah, pelo suporte na avaliação do mercado de vacinas no Brasil e controle de qualidade do estudo.

\section{Referências bibliográficas}

Agência Nacional de Vigilância Sanitária. Lista de Preços de Medicamentos - Preços Fábrica e Máximos de Venda ao Governo. Secretaria Executiva. Câmara de Regulação de Medicamentos (CMED); 2020.

Audsley JM, Tannock GA. Cell-based influenza vaccines: progress to date. Drugs. 2008:68(11):1483-91.

Bacen. Cotações e boletins. Câmbio e capitais internacionais. 2020. Available from: https://www4.bcb.gov.br/pec/taxas/port/ptaxnpesq.asp?frame=1

Barros EN, Cintra O, Rossetto E, Freitas L, Colindres R. Patterns of influenza B circulation in Brazil and its relevance to seasonal vaccine composition. Braz J Infect Dis. 2016;20(1):81-90.

Boikos C, Sylvester GC, Sampalis JS, Mansi JA. Relative Effectiveness of the Cell-Cultured Quadrivalent Influenza Vaccine Compared to Standard, Egg-Derived Quadrivalent Influenza Vaccines in Preventing InfluenzaLike Illness in 2017-2018. Clin Infect Dis. 2020;71(10):e665-71.

Brasil. Análise de materiais - Vacina, Composição: Influenza Trivalente, Tipo: Fragmentada, Inativada, Forma farmacêutica: Suspensão injetável Ano da compra: 2019 - Órgão: Ministério da Saúde. Painel de Preços, 08/05/2020. 2020a. Available from: https://paineldeprecos.planejamento. gov.br/analise-materiais. Accessed on: Jun 4, 2020.

Brasil. BPS - Banco de Preços em Saúde. Brasília: Ministério da Saúde; 2020b. V-3.1.33.

Brasil. Datasus - Departamento de Informática do Sistema Único de Saúde. TABNET - Informações de Saúde. Brasília: Ministério da Saúde; 2019.

Brasil. Diretrizes metodológicas: Diretriz de Avaliação Econômica. Departamento de Ciência e Tecnologia. Brasília: Ministério da Saúde; 2014.

Briggs A, Sculpher M, Claxton K. Decision modelling for health economic evaluation. Oxford: Oxford University Press; 2006.

Cai R, Gerlier L, Eichner M, Schwehm M, Rajaram S, Mould-Quevedo J, et al. Cost-effectiveness of the cell-based quadrivalent versus the standard egg-based quadrivalent influenza vaccine in Germany. J Med Econ. 2021;24(1):490-501.

Canuto V. Avaliação econômica de tecnologias em saúde e limite de custoefetividade. 2012. p. 10, Available from: http://conitec.gov.br/images/ Artigos_Publicacoes/AVE-LimiteCE_VaniaCristinaCanutoSantos.pdf
Demicheli V, Jefferson T, Di Pietrantonj C, Ferroni E, Thorning S, Thomas $R E$, et al. Vaccines for preventing influenza in the elderly. Cochrane Database Syst Rev. 2018a;2(2):CD004876.

Demicheli V, Jefferson T, Ferroni E, Rivetti A, Di Pietrantonj C. Vaccines for preventing influenza in healthy adults. Cochrane Database Syst Rev. 2018b Feb 1;2(2):CD001269.

Dolk C, Eichner M, Welte R, Anastassopoulou A, Van Bellinghen LA, Poulsen Nautrup B, et al. Cost-Utility of Quadrivalent Versus Trivalent Influenza Vaccine in Germany, Using an Individual-Based Dynamic Transmission Model. Pharmacoeconomics. 2016;34(12):1299-308.

Gums JG, Pelletier EM, Blumentals WA. Oseltamivir and influenza-related complications, hospitalization and healthcare expenditure in healthy adults and children. Expert Opin Pharmacother. 2008;9(2):151-61.

IBGE - Instituto Brasileiro de Geografia e Estatística. Contas Nacionais. PIB cresce 1,1\% e fecha 2019 em R\$ 7,3 trilhões, Agência IBGE Notícias. 2020. Available from: https://agenciadenoticias.ibge.gov.br/agencianoticias/2012-agencia-de-noticias/noticias/27007-pib-cresce-1-1-efecha-2019-em-r-7-3-trilhoes

IBGE - Instituto Brasileiro de Geografia e Estatística. Projeção da população por sexo e idades simples, em $1^{\circ}$ de julho - 2010/2060. 2018. Available from: https://www.ibge.gov.br/estatisticas/sociais/populacao/9109projecao-da-populacao.html?=\&t=downloads

INSS. Aposentadoria por Tempo de Contribuição inss.gov.br2018. Available from: https://www.inss.gov.br/beneficios/aposentadoria-por-tempo-decontribuicao/.

Iuliano AD, Roguski KM, Chang HH, Muscatello DJ, Palekar R, Tempia S, et al. Estimates of global seasonal influenza-associated respiratory mortality: a modelling study. Lancet. 2018;391(10127):1285-300.

Jefferson T, Rivetti A, Di Pietrantonj C, Demicheli V. Vaccines for preventing influenza in healthy children. Cochrane Database Syst Rev. 2018;2(2):CD004879.

Karve S, Misurski D, Herrera-Taracena G, Davis KL. Annual all-cause healthcare costs among influenza patients with and without influenza-related complications: analysis of a United States managed care database. Appl Health Econ Health Policy. 2013;11(2):119-28.

McElhaney JE. The unmet need in the elderly: designing new influenza vaccines for older adults. Vaccine. 2005;23 Suppl 1:S10-25.

McGuire PJ, Tarasenko TN, Wang T, Levy E, Zerfas PM, Moran T, et al. Acute metabolic decompensation due to influenza in a mouse model of ornithine transcarbamylase deficiency. Dis Model Mech. 2014;7(2):205-13.

Mertz D, Kim TH, Johnstone J, Lam PP, Science M, Kuster SP, et al. Populations at risk for severe or complicated influenza illness: systematic review and meta-analysis. BMJ. 2013;347:f5061.

Molinari NA, Ortega-Sanchez IR, Messonnier ML, Thompson WW, Wortley PM, Weintraub E, et al. The annual impact of seasonal influenza in the US: measuring disease burden and costs. Vaccine. 2007;25(27):5086-96.

Monteiro N. Em 2020, Ministério da Saúde amplia público para vacinas contra febre amarela e gripe. 2019. Available from: https://bvsms.saude. gov.br/em-2020-ministerio-da-saude-amplia-publico-para-vacinascontra-febre-amarela-e-gripe/

Rajaram S, Suphaphiphat P, van Boxmeer J, Haag M, Leav B, Iheanacho I, et al. Retrospective Assessment of the Antigenic Similarity of Egg-Propagated and Cell Culture-Propagated Reference A/H3N2 Influenza Viruses Compared with Circulating A/H3N2 Viruses Across Influenza Seasons 2002-2003 to 2017-2018. Int J Environ Res Public Health. 2020;17(5433):1-13.

Rothberg MB, Haessler SD. Complications of seasonal and pandemic influenza. Crit Care Med. 2010;38(4 Suppl):e91-7.

Ruiz-Aragón J, Gani R, Márquez S, Alvarez P. Estimated cost-effectiveness and burden of disease associated with quadrivalent cell-based and egg-based influenza vaccines in Spain. Hum Vaccin Immunother. 2020;16(9):2238-44. 
Soespe - Sociedade Espiritossantense de Pediatria. Informe Técnico. Campanha Nacional de Vacinação Contra a Influenza. Brasília; 2014.

SVS - Secretaria de Vigilância em Saúde. Doses de vacina para influenza aplicadas em 2019. Sistema de Informação do Programa Nacional de Imunizações: SI-PNl; 2019a.

SVS - Secretaria de Vigilância em Saúde. Informe epidemiológico Influenza: Monitoramento até a Semana Epidemiológica 52. Boletim Epidemiológico. 2019b. Available from: http://portalsaude.saude.gov.br/ index.php/o-ministerio/principal/leia-mais-o-ministerio/197-secretariasvs/11955-boletins-epidemiologicos-arquivos

SVS - Secretaria de Vigilância em Saúde. Informe Técnico - 21 a Campanha Nacional de Vacinação contra a Influenza. Ministério da Saúde. 2019c. p. 38.
Taveira A, Ely E, Carvalho L, Abdullatif MTGV, et al. Exposição 20 anos de parceria contra a gripe. São Paulo: Instituto Butantan; 2019. p. 1-12.

Van Bellinghen LA, Marijam A, Tannus Branco de Araujo G, Gomez J, Van Vlaenderen I. Cost-utility of quadrivalent versus trivalent influenza vaccine in Brazil - comparison of outcomes from different static model types. Braz J Infect Dis. 2018;22(1):1-10.

WHO - World Health Organization. Immunization, Vaccines and Biologicals - Influenza. Immunization; 2015. Available from: https://www.who.int/ immunization/diseases/influenza/en/.

Zost SJ, Parkhouse K, Gumina ME, Kim K, Diaz Perez S, Wilson PC, et al. Contemporary H3N2 influenza viruses have a glycosylation site that alters binding of antibodies elicited by egg-adapted vaccine strains. Proc Natl Acad Sci U S A. 2017;114(47):12578-83. 